\title{
Homozygosity at the Candida albicans MTL locus associated with azole resistance
}

\author{
Tige R. Rustad, ${ }^{1,2}$ David A. Stevens, ${ }^{3,4}$ Michael A. Pfaller ${ }^{5}$ \\ and Theodore C. White ${ }^{1,2}$
}

Author for correspondence: Theodore C. White. Tel: +1 2062848846 ext. 344. Fax: +1 2062840313.
e-mail: tedwhite@u.washington.edu

1 Department of Pathobiology, School of Public Health and Community Medicine, University of Washington, Seattle, WA 98195, USA

2 Seattle Biomedical Research Institute, 4 Nickerson St Suite 200, Seattle, WA 98109-1651, USA

3 Department of Medicine, Santa Clara Valley Medical Center and California Institute for Medical Research, San Jose, CA 95128, USA

4 Department of Medicine, Division of Infectious Diseases and Geographic Medicine, Stanford University, Stanford, CA 52242, USA

5 Department of Pathology, University of lowa, lowa City, IA 52242, USA

\begin{abstract}
Antifungal drug resistance in the pathogenic fungus Candida albicans is a serious threat to the growing population of immunocompromised patients. This study describes a significant correlation between loss of heterozygosity at the $C$. albicans mating-type-like (MTL) locus and resistance to azole antifungals. A pool of $\mathbf{9 6}$ clinical isolates consisting of $\mathbf{5 0}$ azole-resistant or susceptible dose-dependent isolates and 46 azole-susceptible isolates was screened by PCR for the presence of MTLa1 and MTL $\alpha 1$. These genes were used as markers for the MTLa and MTL $\alpha$ loci. Both loci were present in 84 of the isolates. Six isolates failed to amplify MTLa1 and six failed to amplify MTL $\alpha 1$. Further PCR analysis demonstrated that loss of the MTLa1 and MTL $\alpha 1$ genes corresponded to loss of all of the loci-specific genes, resulting in homozygosity at the MTL locus. Southern analysis and single nucleotide polymorphism (SNP) analysis were used to determine that this loss of heterogeneity was due to replacement of one of the MTL loci with a duplicate of the other locus resulting in two homozygous copies of the MTL locus. Of the 12 homozygous isolates, one isolate was sensitive to azole drugs. Statistical analysis of the data demonstrates a strong correlation between homozygosity at the MTL locus and azole resistance $(P<0.003)$. In a set of serial isolates, an increase in azole resistance correlated with the loss of heterozygosity at the MTL locus, lending further strength to the correlation. Gene disruptions of the MTL loci were found to have no effect on azole susceptibility.
\end{abstract}

Keywords: pathogenic fungi, mating locus, drug resistance

\section{INTRODUCTION}

Candida albicans is a pathogenic fungus responsible for frequent, recurrent oral and vaginal candidiasis in AIDS patients, and systemic infections in transplant patients (Odds, 1988). C. albicans is also the fourth most common nosocomial bloodstream isolate (Pfaller et al., 1998). Untreated, systemic candidiasis has a mortality rate of $\sim 40 \%$ (Wright \& Wenzel, 1997). Treatment options are limited to the toxic but fungicidal polyene drugs and the fungistatic azole drugs. Prophylactic use of the azole drugs, combined with their fungistatic nature, has resulted in widespread azole resistance (Georgopapadakou \& Walsh, 1996; Law et al., 1994).

Abbreviations: $\mathrm{CHEF}$, contour-clamped homogeneous electric field; chr, chromosome; EtBr, ethidium bromide; SNP, single nucleotide polymorphism.
A number of mechanisms have been correlated with azole resistance, including mutation and overexpression of the gene encoding the azole target enzyme, ERG11, and overexpression of the genes encoding the efflux pumps MDR1, CDR1 and CDR2 (White et al., 1998). However, there remains a significant portion of resistant clinical isolates in which none of the resistance mechanisms previously described can be identified (Stevens \& White, 2000).

The C. albicans mating-type-like (MTL) loci are homologous to the Saccharomyces cerevisiae mating type (MAT) loci. The MAT loci contain the transcriptional regulators MATa1, MAT $\alpha 1$, and MAT $\alpha 2$ that determine the mating type (MATa or MAT $\alpha$ ) of haploid S. cerevisiae strains (Johnson, 1995; Herskowitz et al., 1997). In haploid MATa strains, the MATa1 gene product has no known function. In haploid MAT $\alpha$ strains, Mat $\alpha 1 \mathrm{p}$ acts as an activator of the $\alpha$ mating-type 

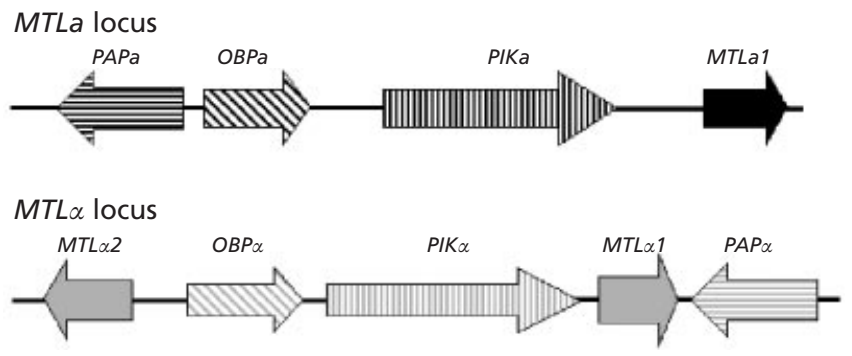

Fig. 1. Map of the $C$. albicans mating-type-like (MTL) loci Genes specific to the MTL locus include those encoding poly(A) polymerase $(P A P)$, oxysterol-binding protein $(O B P)$, and phosphoinositol kinase $(P I K)$, and the mating-type homologues MTLa1, MTL $\alpha 1$ and MTL $\alpha 2$. The a and $\alpha$ versions of PAP, PIK and $O B P$ have approximately $60 \%$ homology to each other. The MTL genes have no significant homology to each other.

specific genes and Mat $\alpha 2 \mathrm{p}$ inhibits mating-type $a$ specific genes. In diploid $S$. cerevisiae, both the MATa and $M A T \alpha$ loci are present. In these diploid strains Mata1p and Mat $\alpha 2 p$ form a heterodimer that acts to repress haploid-specific genes.

The C. albicans MTL loci contain genes homologous to MATa1, MAT $\alpha 1$ and MAT $\alpha 2$ in the same genomic arrangement as the $S$. cerevisiae genes (Hull \& Johnson, 1999). However, the C. albicans loci also contain genes unique to fungal mating loci, including genes encoding an oxysterol-binding protein $(O B P)$, a poly $(A)$ polymerase $(P A P)$, and a phosphoinositol kinase $(P I K)$ (Fig. 1). A copy of each of these three genes is present in both the MTL $a$ locus and the MTL $\alpha$ locus. The $a$ and $\alpha$ copies of each of the three genes have only $60 \%$ identity to each other, suggesting that homologous recombination within the MTL loci is not common, unlike the rest of the genome (Hull \& Johnson, 1999). The MTL loci span approximately $9 \mathrm{~kb}$ and are flanked on both sides by identical sequence, suggesting that the flanking regions undergo homologous recombination like the rest of the genome (Hull \& Johnson, 1999). Most wild-type C. albicans strains are diploid and have both the MTLa and $M T L \alpha$ loci (Magee \& Magee, 2000).

The gene encoding the target enzyme for azole drugs, ERG11, is located on chromosome (chr) 5, the same chromosome as the MTL loci. Previous work has demonstrated that gene conversion or mitotic recombination associated with the ERG11 gene is associated with azole resistance (White, 1997a). Therefore, it was of interest to determine if these gene conversions or mitotic recombinations might have affected the $M T L$ loci.

The current study describes a screen of clinical isolates to determine their genotype at the MTL locus. That screen demonstrated a significant correlation between homozygosity at the C. albicans MTL locus and resistance to the antifungal fluconazole. Southern analysis was used to confirm the PCR results and to demonstrate the presence of duplicate copies of the remaining MTL locus in the MTL homozygous strains.

\section{METHODS}

Strains and growth of cultures. Fifty of the clinical isolates were sent to M.A.P. from locations around the USA for further characterization. Thirty-six of the clinical isolates were sent to D.A.S. from California locations (Stevens \& White, 2000). Ten isolates were sent to T.C.W. from California locations. Isolates were plated on ChromAgar (DRG International) to characterize them as C. albicans. The gene disruptions were provided by Christina Hull (Duke University, Durham, NC, USA) and were constructed in the laboratory of Alexander Johnson (University of California, San Francisco) (Hull et al., 2000). The FH serial isolates used in this study were described previously (Marr et al., 1997, 1998). Laboratory strains 3153A (ATCC 28367, American Type Culture Collection), SC5314 (Fonzi \& Irwin, 1993) and CAI4 (Fonzi \& Irwin, 1993) were used as controls. The isolates were stored as frozen stocks kept at $-70{ }^{\circ} \mathrm{C}$ in YEPD (10 g yeast extract, $20 \mathrm{~g}$ peptone, $20 \mathrm{~g}$ glucose per litre) with $10 \%(\mathrm{v} / \mathrm{v})$ glycerol. The isolates were struck from frozen stocks onto YEPD plates and grown at $30^{\circ} \mathrm{C}$ overnight. Plates were kept at $4{ }^{\circ} \mathrm{C}$ and subcultured from single colonies every 2 weeks. Single-colony inocula were grown in YEPD liquid medium for DNA and CHEF gel block preparation.

Susceptibility testing. Fluconazole MICs were determined by broth microdilution or macrodilution using the standardized NCCLS protocol (National Committee for Clinical Laboratory Standards, 1997) as described previously (Stevens \& White, 2000). Confirmation of the MICs for the MTL homozygous strains was done using E-test strips according to the manufacturer's instructions (AB Biodisk, Solna, Sweden).

PCR analysis. Oligonucleotides used for PCR in this study are listed in Table 1 . PCR was performed in $50 \mu$ l reactions containing: $1 \times$ PCR buffer $(50 \mathrm{mM} \mathrm{KCl}, 10 \mathrm{mM}$ Tris $/ \mathrm{HCl}$, pH 8.3), 2.5 mM $\mathrm{MgCl}_{2}, 200 \mu \mathrm{M}$ dNTPs, 100 ng template DNA ml ${ }^{-1}, 20 \mu \mathrm{M}$ each primer, and $5 \mathrm{U}$ Taq polymerase. PCR was performed under the following conditions in a PTC-100 programmable thermal controller (MJ Research): denaturing at $94^{\circ} \mathrm{C}$ for $10 \mathrm{~min}$ followed by 30 cycles of $1 \mathrm{~min}$ denaturing at $94^{\circ} \mathrm{C}, 2 \mathrm{~min}$ annealing at $55^{\circ} \mathrm{C}$, and $3 \mathrm{~min}$ elongation at $72{ }^{\circ} \mathrm{C}$, followed by a final extension step at $72^{\circ} \mathrm{C}$ for $10 \mathrm{~min}$. PCR fragments were visualized by electrophoresis on a $1.2 \%$ agarose gel in $1 \times$ TBE $(0.089 \mathrm{M}$ Tris/borate, $0.089 \mathrm{M}$ boric acid, $2 \mathrm{mM}$ EDTA) at $100 \mathrm{~V}$ for $1 \mathrm{~h}$ and stained with ethidium bromide (EtBr).

Statistical analysis. Freely available on-line software was used to calculate a $P$-value using Fisher's exact test (Uitenbroek, 1997).

CHEF (contour-clamped homogeneous electric field) gels. Gel blocks for CHEF gels were made using the following method (adapted from the method posted at http://alces.med.umn. edu/candida/methods.html). From overnight cultures, $2 \times 10^{8}$ cells were removed and washed twice in $50 \mathrm{mM}$ EDTA $\mathrm{pH} 7 \cdot 5$. Cells were resuspended in $200 \mu \mathrm{l}$ EDTA pH $7 \cdot 5$ and treated with $15 \mu \mathrm{l}$ Zymolyase solution $(50 \%$, v/v, glycerol, $2 \cdot 5 \mathrm{mg}$ Zymolyase $\mathrm{ml}^{-1}, 10 \mathrm{mM}$ sodium phosphate $\mathrm{pH} 7 \cdot 5$ ). An equal volume of $1.5 \%$ low-melting-point agarose in $1 \times$ TAE (4 mM Tris/acetate, $1 \mathrm{mM}$ EDTA pH 8) was added and the mixture poured into moulds. Blocks were allowed to cool at $4{ }^{\circ} \mathrm{C}$ for $30 \mathrm{~min}$ and were then added to a solution of 
Table 1. Oligonucleotides used for PCR

\begin{tabular}{|c|c|c|c|c|}
\hline Gene or locus & Reference* & Location $\dagger$ & Size (bases) & Sequence $\left(5^{\prime}-3^{\prime}\right)$ \\
\hline \multirow[t]{2}{*}{ MTLa1 } & 1 & $9783-10318$ & 535 & 1. TAAGAATGAAGACAACGAGG \\
\hline & & & & 2. CGTGTTTTTCTGCTATCAATTCC \\
\hline \multirow[t]{2}{*}{$M T L \alpha 1$} & 1 & $7999-8421$ & 423 & 1. TACATTCTGGTCGCGATGCTC \\
\hline & & & & 2. GTAATCCAAAGCCTCGCATAA \\
\hline \multirow[t]{2}{*}{$M T L \alpha 2$} & 1 & $1939-2553$ & 615 & 1. ATGAATTCACATCTGGAGGC \\
\hline & & & & 2. CTGTTAATAGCAAAGCAGCC \\
\hline \multirow[t]{2}{*}{$\mathrm{OBPa}$} & 1 & $3976-5517$ & 1551 & 1. AATTGCTGGTCGCTGATCG \\
\hline & & & & 2. ATTATTCCCAATGTGTGCCAAC \\
\hline \multirow[t]{2}{*}{$O B P \alpha$} & 1 & $3207-4498$ & 1291 & 1. AATTTATCCAGCGAACATGCAC \\
\hline & & & & 2. CTTCTGTCCTGGAACAATCGG \\
\hline \multirow[t]{2}{*}{$P A P a$} & 1 & $1674-3680$ & 2007 & 1. AATCAAGCATACGGTGTTACAC \\
\hline & & & & 2. CCTCATGTCGCCAACCACAG \\
\hline \multirow[t]{2}{*}{$P A P \alpha$} & 1 & $8394-10205$ & 1812 & 1. GACTGGTTATGCGAGGCTTTG \\
\hline & & & & 2. AGATCCTGGACCATAGACTCC \\
\hline \multirow[t]{2}{*}{ PDE1 $\neq$} & 2 & $800-1151$ & 352 & 1. TAATATGCTAGGTGGGGGTTCCTT \\
\hline & & & & 2. GCCCAATATGCCTAGTTTCAAAATC \\
\hline \multirow[t]{2}{*}{$A R G 4 \ddagger$} & 2 & $1698-1893$ & 196 & 1. TCACGGCAATTCTTGAACGAG \\
\hline & & & & 2. GCTAAAGCACCAGATCCTAATGGAG \\
\hline \multirow[t]{2}{*}{$\mathrm{C} 2 \mathrm{~F} 7 \ddagger$} & 2 & $1-282$ & 282 & 1. GTTTGATCTGGAACGATCTC \\
\hline & & & & 2. AGAAACCAACCAGCGTCTTC \\
\hline \multirow[t]{2}{*}{ ERG11‡ } & 3 & $133-682$ & 549 & 1. AGATCATAACTCAATATG \\
\hline & & & & 2. ACCCCATGAGTTTTTCTTT \\
\hline \multirow[t]{2}{*}{ ERG11 term\$ } & 3,4 & 1495,525 & 585 & 1. GGGAAAGTTTCTAAAGGGG \\
\hline & & & & 2. GAAGTAACGCTCATATG \\
\hline \multirow{4}{*}{$\begin{array}{l}\text { MTLa-ERG11 } \\
-M T L \alpha\end{array}$} & 5 & & 150 & 1. TCGCAAGCAGGAAGAAGAAGAAGCTTTCATTTTTCCC- \\
\hline & & & & ACAGTAAG TACCG GGTGGTCAACATACTTCTGC \\
\hline & & & & 2. CTATTTCCAATCCGGAACACCAACGAGAACTATCATC- \\
\hline & & & & TAAATTAGCGGAT ACCTAAATGTAACAAGAACC \\
\hline
\end{tabular}

*1, Hull \& Johnson (1999); 2, Cowen et al. (1999); 3, Lai \& Kirsch (1989); 4, White (1997b); 5, this study.

†Locations are based on the published sequence as referenced in the previous column.

‡Fragments used for SNP analysis. PDE1 digested with Tru9I, ARG4 with HaeIII, C2F7 with DdeI, ERG11 with HindIII.

$\$ S N P s$ in the ERG11 terminator region detected by sequence analysis.

0.5 M EDTA, $10 \mathrm{mM}$ Tris/ $\mathrm{HCl} \mathrm{pH} 7 \cdot 5$, and incubated overnight at $30^{\circ} \mathrm{C}$. Blocks were then washed three times in $0.5 \mathrm{M}$ EDTA pH 9.5, treated with $2 \mathrm{ml}$ of a lysis solution $(2.5 \mathrm{mg}$ proteinase $\mathrm{K} \mathrm{ml}^{-1}$, $1 \% \mathrm{~N}$-lauroylsarcosine, $0.5 \mathrm{M}$ EDTA, $10 \mathrm{mM}$ Tris $/ \mathrm{HCl} \mathrm{pH} \mathrm{9.5)} \mathrm{and} \mathrm{incubated} \mathrm{overnight} \mathrm{at} 50{ }^{\circ} \mathrm{C}$. Blocks were washed three times with $50 \mathrm{mM}$ EDTA pH 7.5 and stored for up to 3 months at $4{ }^{\circ} \mathrm{C}$ in $50 \mathrm{mM}$ EDTA pH 7.5. CHEF electrophoresis was done using a Bio-Rad CHEF Mapper with the following conditions: $0.8 \%$ agarose gel run at $14{ }^{\circ} \mathrm{C}$ at $3 \mathrm{~V} \mathrm{~cm}^{-1}$ with a 106 degree angle in $1 \times$ TAE. Running time was $50 \mathrm{~h}$ with a 2-8 min linear ramp. CHEF gels were stained with EtBr and photographed using an EagleEye II Still Video System (Stratagene). Band intensities were quantified using ImageQuant software (Molecular Dynamics).

Southern blots. CHEF gels were treated with $0 \cdot 1 \mathrm{M} \mathrm{HCl}$ for 15 min followed by three washes in water. Southern blots were then performed using standard techniques (Sambrook et al., 1989). Probes for labelling Southern blots were made using Prime-a-gene kits (Promega) or kinase labelling (Sambrook et al., 1989). PCR products amplified from CAI4 DNA using the primers listed in Table 1 were used as probes for MTLa1, $M T L \alpha 1, O B P a, O B P \alpha$ and $M T L \alpha 2$. The triple probe (MTLa1ERG11-MTL $\alpha 1$ ) was constructed by using two oligonucleo- tides containing $50 \mathrm{bp}$ tails homologous to one of the MTL genes and $20 \mathrm{bp}$ heads homologous to a region of the ERG11 gene to amplify a $150 \mathrm{bp}$ double-stranded fragment that contained 50 bp of MTLa1, ERG11 and MTL $\alpha 1$. This fragment was radiolabelled and used to probe for those genes. Southern blots were scanned using a STORM phosphoimager (Molecular Dynamics) and quantified using ImageQuant software according to the manufacturer's instructions.

Single nucleotide polymorphism (SNP) analysis. PCR fragments from chr 5, chr 6 and chr 7 (PDE1, ARG4 and C2F7 from Table 1) were amplified from the MTL homozygous strains as previously described (Cowen et al., 1999). These fragments were then digested with the appropriate restriction enzyme (Table 1 ) in $40 \mu \mathrm{l}$ reactions containing $1 \times$ reaction buffer, $10 \mu \mathrm{g}$ PCR DNA, and $1 \mu \mathrm{l}$ restriction enzyme incubated overnight at $37^{\circ} \mathrm{C}$. Digestion products were then electrophoresed in $1.5 \%$ agarose in $1 \times \mathrm{TBE}$ at $100 \mathrm{~V}$ for $1 \mathrm{~h}$ with EtBr.

The terminator region of ERG11 on chr 5 was amplified using the primers listed in Table 1 and the conditions used to amplify the SNP fragments. This PCR fragment was sequenced (Marr et al., 1998) using the same primers as used for PCR and screened for heterozygous polymorphisms visible as over- 
lapping peaks on the sequencing read. Heterozygous polymorphisms were confirmed from the sequence of the complementary strand of the PCR fragment.

\section{RESULTS}

\section{PCR screen of the MTL loci of clinical isolates of} C. albicans

Ninety-six clinical isolates were selected for analysis; fifty of these isolates were resistant or susceptible dose-dependent to fluconazole with a MIC greater than
$8 \mu \mathrm{g} \mathrm{ml}^{-1}$. Oligonucleotides were designed to specifically amplify the three MTL genes: MTLa1, MTL $\alpha 1$ and MTL $\alpha 2$ (Table 1). PCR was performed with the MTLa1and MTL $\alpha 1$-specific oligonucleotides on genomic DNA from each of the isolates. PCR fragments from MTLa1 and MTL $\alpha 1$ were generated from 84 of the 96 isolates screened. Genomic DNA from six of the isolates did not amplify MTLa1; DNA from six unrelated isolates did not amplify $M T L \alpha 1$ (Fig. 2). Of these 12 isolates homozygous at the MTL locus (MTLhom) only one was a susceptible isolate (Table 2). These PCR results were
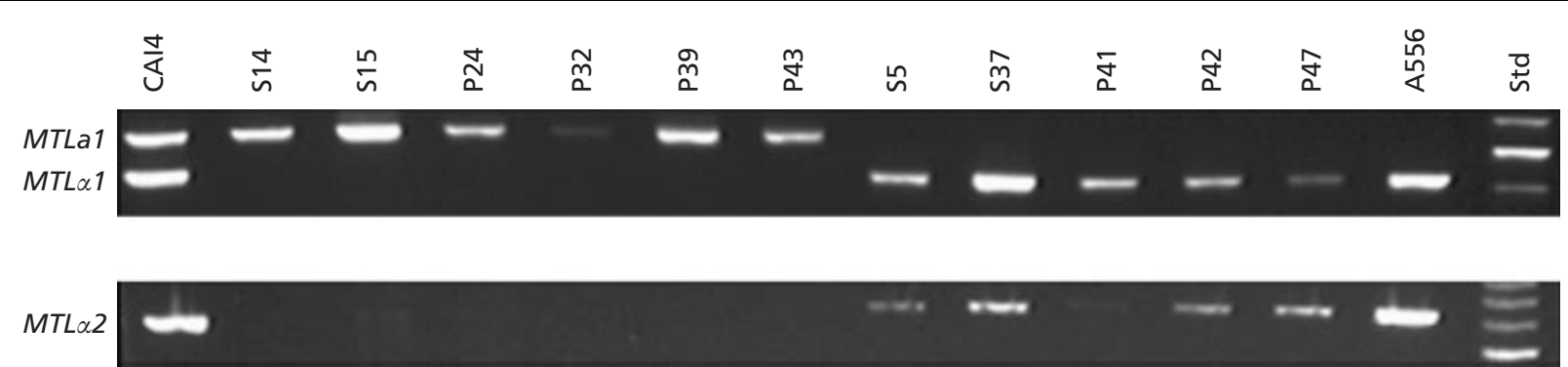

Fig. 2. $P C R$ screen of $M T L$ genotype in clinical isolates. DNA fragments from within the $M T L a 1, M T L \alpha 1$ and $M T L \alpha 2$ genes were amplified using genomic DNA from 96 clinical isolates and visualized on EtBr-stained agarose gels. Reactions where one of the two PCR fragments failed to amplify were repeated. CAI4 was used as a MTLaIMTL $\alpha$ control strain. Twelve isolates failed to amplify one of the two fragments. Strains in which the MTL $\alpha 1$ fragment failed to amplify correspondingly failed to amplify a fragment from $M T L \alpha 2$. Std, size standards.

Table 2. MTL genotype, fluconazole MIC and SNP analysis of heterozygosity for selected strains

\begin{tabular}{|lccc|}
\hline Strain & MTL genotype* & Fluconazole MIC $\dagger$ & $\begin{array}{c}\text { Chromosome(s) with } \\
\text { heterozygous SNPs } \neq\end{array}$ \\
\hline S14 & $a$ & $>64$ & 5 \\
S15 & $a$ & $>64$ & 7 \\
P24 & $a$ & $0 \cdot 038$ & 7 \\
P32 & $a$ & $>64$ & - \\
P39 & $a$ & $>64$ & 7 \\
P43 & $a$ & $>64$ & 5 \\
S5 & $\alpha$ & $>64$ & 5 \\
S37 & $\alpha$ & 16 & 5 \\
P41 & $\alpha$ & 16 & 7 \\
P42 & $\alpha$ & $>64$ & - \\
P47 & $\alpha$ & $>64$ & 7 \\
A556 & $\alpha$ & $0 \cdot 5$ & 5 \\
S1 & $a / \alpha$ & 32 & 5 \\
S2 & $a / \alpha$ & $0 \cdot 125$ & 5 \\
$\# 1$ & 2 & 5 \\
CAI4 & $a / \alpha$ & $0 \cdot 125$ & - \\
3153A & $a / \alpha$ & 4 & 5 \\
FH 1 & $a / \alpha$ & $>64$ & 5 \\
FH 5 & $a / \alpha$ & $\alpha$ &
\end{tabular}

*Determined by PCR amplification of the MTLa1 and MTL $\alpha 1$ genes.

† MIC determined by Etest.

‡Chromosomes 5, 6, and 7 were screened for the presence of heterozygous SNPs (Cowen et al., 1999).

SSNPs detected by sequencing of ERG11 terminator region. 


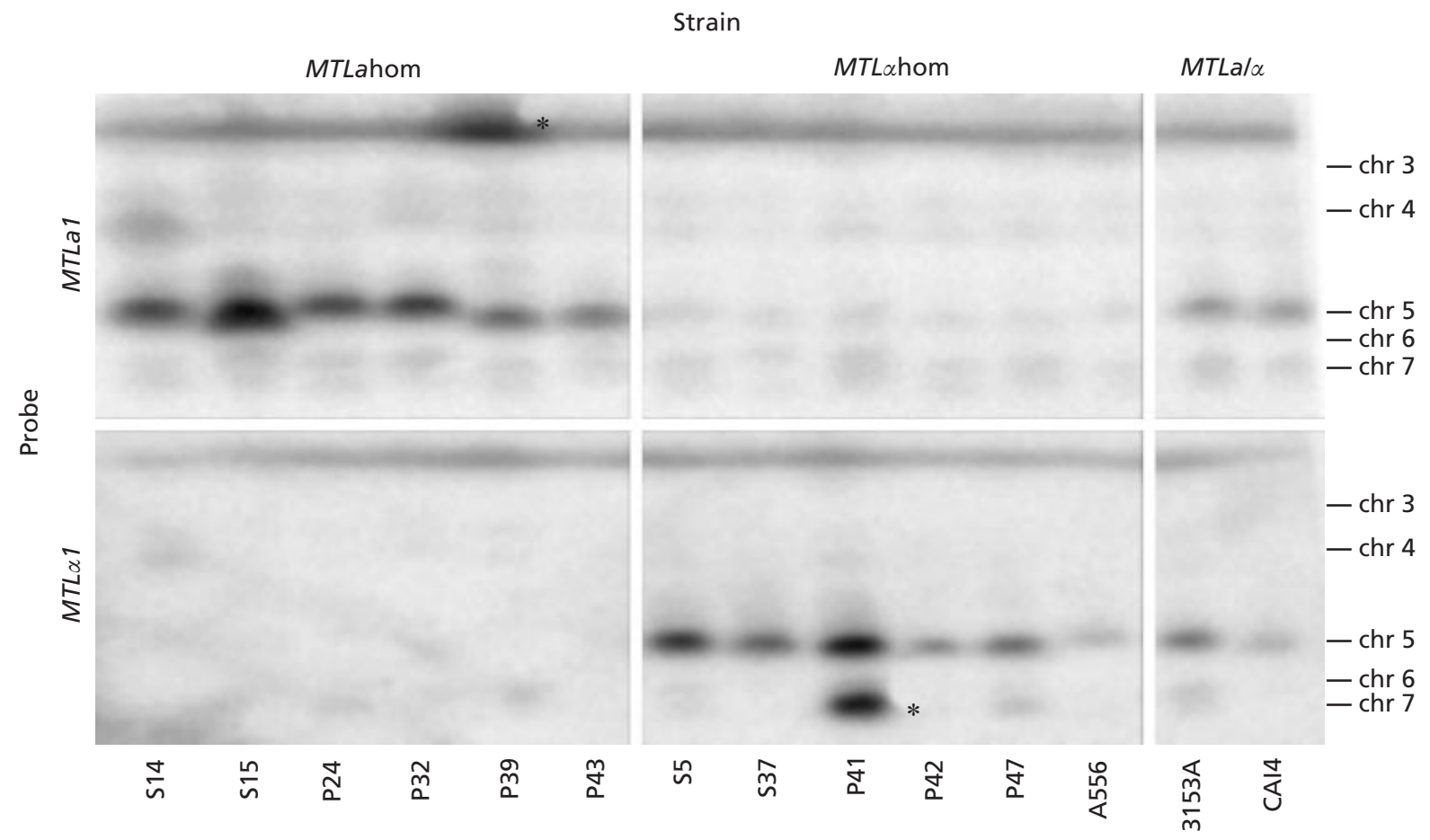

Fig. 3. Southern blot analysis using radiolabelled MTLa1 and MTL $\alpha 1$. Genomic DNA from the $M T L$ hom isolates and two control strains (3153A and CAI4) was separated into chromosomal bands by CHEF electrophoresis and blotted onto nitrocellulose paper. The blot was then hybridized to radiolabelled PCR fragments of MTLa1 and MTL $\alpha 1$ and analysed on a phosphoimager. The MTLa1 and MTL $\alpha 1$ probes selectively hybridized to chr 5 of the MTLahom and MTLahom respectively, and both probes hybridized to chr 5 of the control strains. Asterisks denote possible extra copies of the MTLA1 and MTL 1 genes on chromosomes other than chr 5.

confirmed by hybridization of $M T L$-specific probes to Southern blots of CHEF gels, which resolve the chromosomes as distinct bands on the gel (Fig. 3). Based on hybridization, strains P39 and P41 appear to have extra copies of MTLa1 and MTL $\alpha 1$ on other chromosomes. The presence of only one of the two MTL genes in these 12 isolates, as determined by PCR and Southern blot hybridization, suggests that these strains are homozygous at the MTL locus.

In order to determine the extent of the loss of heterogeneity, the presence of $M T L \alpha 2, O B P a, O B P \alpha, P A P a$ and $P A P \alpha$ was determined by PCR in the 12 MTLhom strains. The six strains missing MTL $\alpha 1$ (MTLahom) were found to also lack $M T L \alpha 2$. In addition, the $O B P \alpha$ and $P A P \alpha$ genes were not present in these MTLahom strains. Conversely, the $\mathrm{OBP} a$ and $P A P a$ genes were not present in the MTL $\alpha$ hom strains (data not shown). Neither $\mathrm{OBPa}$ nor $\mathrm{OBP} \alpha$ could be amplified from strain P32, which is MTLahom. However, a probe specific for $\mathrm{OBP} a$ hybridized to genomic DNA from strain P32 in a Southern blot (data not shown). Neither PAPa nor PAP $\alpha$ could be amplified from strain P41, which is MTL $\alpha$ hom. However, a probe specific for $P A P \alpha$ hybridized to genomic DNA from strain P41 (data not shown). The fact that the loci-specific $O B P, P A P$ and $M T L$ genes cosegregate in these 12 MTLhom strains indicates that
Table 3. Statistical analysis of the relationship between MTL genotype and resistance

The $P$ value calculated using Fisher's exact test (Uitenbroek, 1997) was $0 \cdot 003$.

\begin{tabular}{|lccc|}
\hline & MTLa/ $\boldsymbol{\alpha}$ & $\begin{array}{c}\text { MTLa or } \\
\text { MTL } \alpha \dagger\end{array}$ & Total \\
\hline Sensitive* $^{*}$ & 45 & 1 & 46 \\
Resistant $^{*}$ & 39 & 11 & 50 \\
Total & 84 & 12 & 96 \\
\hline
\end{tabular}

*Sensitive, fluconazole $\mathrm{MIC}<8 \mu \mathrm{g} \mathrm{ml}^{-1}$; resistant, fluconazole $\mathrm{MIC} \geqslant 8 \mu \mathrm{g} \mathrm{ml}^{-1}$.

† MTL genotype determined by PCR amplification.

the loss of heterozygosity extends through the entire $9 \mathrm{~kb} M T L$ locus in these strains.

Statistical analysis of the 96 strains (Table 3 ) indicates a strong correlation between homozygosity at the MTL locus and azole resistance (Fisher exact, $P<0 \cdot 0029$ ) (Uitenbroek, 1997). The equal distribution of MTLhom isolates, with six missing MTLa1 and six missing 


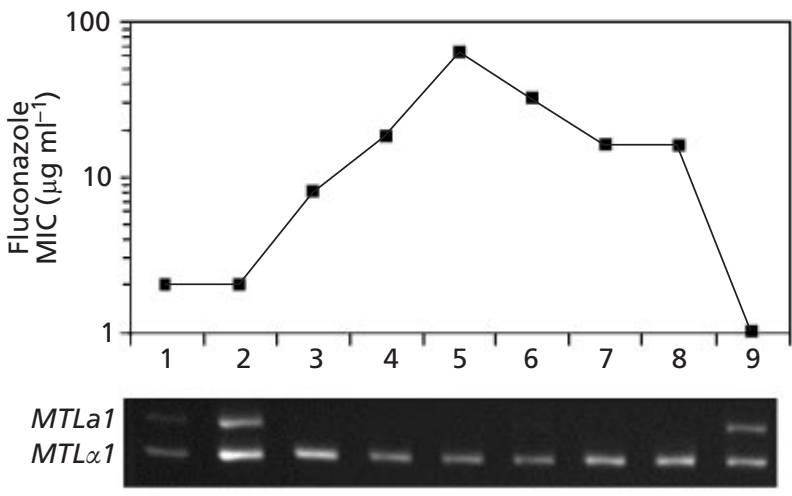

Fig. 4. $M T L$ genotype and fluconazole susceptibility of a serial set of transiently resistant clinical isolates (FH1 to FH9). Fluconazole MICs for the series of nine isolates were determined using the NCCLS macrodilution method. PCR was used to amplify fragments from MTLa1 and MTL $\alpha 1$, which were visualized on EtBr-stained agarose gels. MTLa1 failed to amplify in FH3 to FH8, corresponding to an increase in the fluconazole MIC. The final isolate in the series is sensitive and amplified both MTLa1 and MTL $\alpha 1$.

$M T L \alpha 1$, suggests that homozygosity at either locus is associated with resistance.

\section{Loss of $M T L$ heterozygosity in a set of serial isolates}

This laboratory has previously studied several matched sets of susceptible and resistant clinical isolates (Marr et al., 1997; White, 1997a, b; White et al., 1997), including sets from AIDS patients and sets from bone marrow transplant patients. Each set of isolates develops azole resistance from susceptibility during the course of the series. Two of these sets have an increase in the expression of the CDR1-encoded ATP-binding cassette pump correlated with the reduced susceptibility to fluconazole. One of these sets sequentially gained all of the known correlates of resistance, including CDR1 overexpression. These four sets were assessed for the presence of the MTL loci using the methods described above. In three of these sets, every isolate was heterozygous at the MTL locus (data not shown).

However, in one of these series $(\mathrm{FH})$ a switch to a homozygous $M T L \alpha$ locus corresponded to the first significant increase in fluconazole resistance (Fig. 4). This increase had previously been correlated with an increase in the expression of CDR1 (Marr et al., 1997). The final isolate in this series is sensitive to fluconazole and is also heterozygous at the MTL locus (Fig. 4) (Marr et al., 1997). It is possible to induce azole resistance in vitro in the first sensitive isolate of this series by serially transferring the isolate in the presence of fluconazole (Marr et al., 1998). Several of the resulting resistant isolates, induced to resistance in vitro, are also homozygous $M T L \alpha$ (data not shown). This correlation provides further evidence of a link between loss of $M T L$ heterogeneity and azole resistance.

\section{Polyploidy of chromosome 5 in MTLhom strains}

Homozygosity at the MTL locus could be the result of shedding one copy of chr 5, which carries the MTL loci. The chromosomes of the 12 strains were analysed using CHEF electrophoresis to determine how many copies of chr 5 are present. The chromosomes of the MTLhom isolates were separated on a CHEF gel, then stained with ethidium bromide, visualized using a digital camera (Fig. 5a) and quantified. For each strain, the intensities of chr 5 and chr 6 were normalized to the intensity of chr 4 (Fig. 5b). Since chr 5 and chr 6 are smaller than chr 4, it is expected that the staining of chr 5 and $\operatorname{chr} 6$ with EtBr will be slightly reduced compared with chr 4 . With the exception of strains P39 and S14, the intensities of the chr 5 and chr 6 bands were as intense as the chr 4 band. Chr 5 and 6 of strain P39 stained with half the intensity of chr 4, suggesting that there are either additional copies of chr 4 in this strain or there are only single copies of chr 5 and 6. Chr 6 in strain S14 stained with half the intensity of chr 4 and chr 5 , indicating that S14 has only one copy of chr 6 . These twofold differences were reproduced in three independent experiments. The equal intensity of the chr 4, chr 5 and chr 6 bands indicates that there are roughly equivalent amounts of these chromosomes for most strains. Thus the MTLhom strains are not aneuploid for chr 5.

\section{SNP analysis of MTLhom strains}

Based on the CHEF chromosome quantification, it is possible that the MTLhom strains are euploid and haploid, rather than diploid. Although there is some evidence to indicate the existence of meiosis in $C$. albicans, haploid strains of C. albicans have not been previously described (Lott \& Effat, 2001). However, the MTL locus may provide a new marker for detecting haploid strains. To assess the ploidy of the MTLhom strains, we screened for heterozygous single nucleotide polymorphisms (SNPs).

In these analyses, a PCR fragment is amplified and digested with appropriate restriction enzymes. One of the two alternative sequences at these SNP locations contains a restriction site, the other sequence does not. This allows for rapid screening of sequence differences without direct sequencing. Short stretches of DNA from genes on chr 5 (ERG11, PDE1), chr 6 (locus C2F7), and chr 7 (ARG4) containing known SNPs (Cowen et al., 1999) were analysed in this way. Heterozygosity was detected by the presence of both digested and undigested bands, and would indicate the presence of at least two copies of the chromosome carrying the SNP. Homozygosity could result from either homozygous multiple copies or a single copy of the SNP. In 8 of the 12 MTLhom isolates at least one heterozygous SNP was found (Table 2). Three of these heterozygous SNPs were on $\operatorname{chr} 5$.

In addition to PCR, sequencing and Southern analysis were used to detect heterozygous SNPs. Sequencing of the noncoding region between ERG11 and THR1 

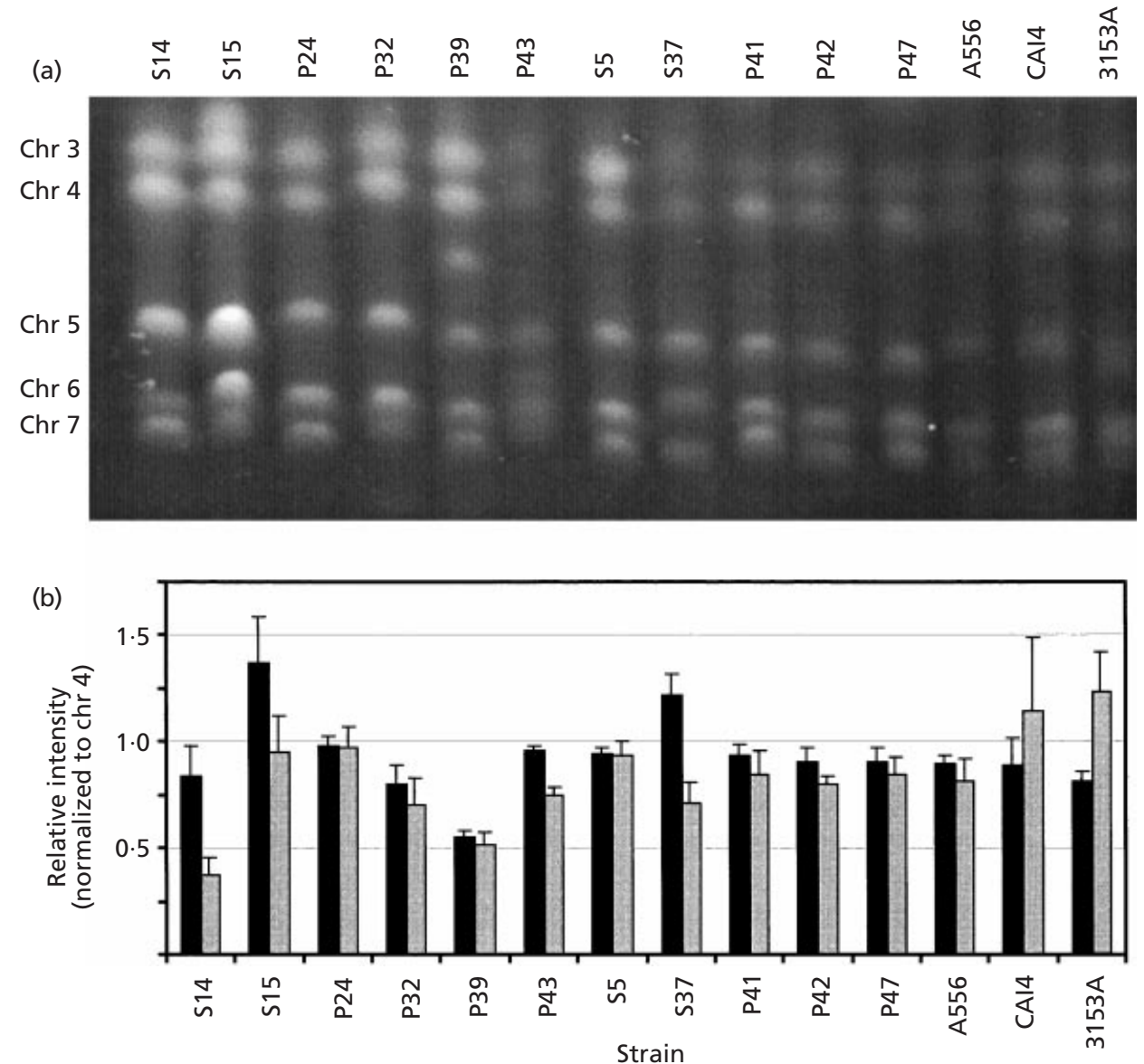

Fig. 5. Quantification of chromosomal bands from an EtBr-stained CHEF gel. (a) Genomic DNA was separated into chromosomal bands using CHEF electrophoresis. (b) A digital image of the EtBr-stained gel was quantified using ImageQuant software. The intensity of chr 5 (dark bars) and chr 6 (light bars) was normalized to the intensity of chr 4 from the same strain. Bars show the mean of three experiments with standard deviation. Chrs 5 and 6 stained with slightly less intensity compared to chr 4 for most of the isolates analysed. Isolate S14 appears to have a single copy of chr 6. Both chr 5 and chr 6 stained with half the intensity of chr 4 in isolate P39.

revealed heterozygosity on chr 5 for two additional isolates. The heterozygosity of the ERG11 bands in Fig. $6(\mathrm{a})$ is another form of SNP analysis demonstrating that three of the 12 MTLhom strains are diploid at the ERG11 locus. The heterozygosity of the ERG11 bands in Fig. 6(a) was only seen in strains already known to have other heterogeneous SNPs on chr 5. A total of 5 of the 12 MTLhom strains have heterogeneity of chr 5, and 10 of the 12 strains have some known heterogeneity. This suggests that most of these MTLhom strains are not haploid and provides further evidence that at least five of these strains carry two copies of chr 5 .

\section{Two copies of the MTL locus are present in MTLhom strains}

Another possible mechanism to explain MTL homozygosity is deletion of one MTL locus and subsequent joining of sequences flanking the MTL locus. Southern analysis was used to detect the copy number of the remaining MTL locus in the MTLhom strains. The relative intensities of gene-specific fragments, detected as bands on Southern blots probed with locus-specific probes, were used to determine the number of $M T L$ loci present in the MTLhom strains. Normally, three separate probes would be used for each gene (MTLa1, MTL $\alpha 1$ and a control such as ERG11). However, multiple probes would introduce variability due to differences in specific activity and binding efficiency of the probes. To reduce this variability a $150 \mathrm{bp}$ oligonucleotide consisting of $50 \mathrm{bp}$ pieces from MTLa1, MTL 1 and ERG11 was constructed using PCR (Table 1). This oligonucleotide was radiolabelled and used to probe a Southern blot of HaeIII-digested genomic DNA from the 12 MTLhom strains and 6 MTL heterozygous (MTLhet) controls (Fig. 6a).

The MTLa1 and MTL $\alpha 1$ bands were easily identified by their absence in the MTLhom strains. The two different higher molecular mass bands are differently sized restriction fragments of ERG11 caused by disruption of 

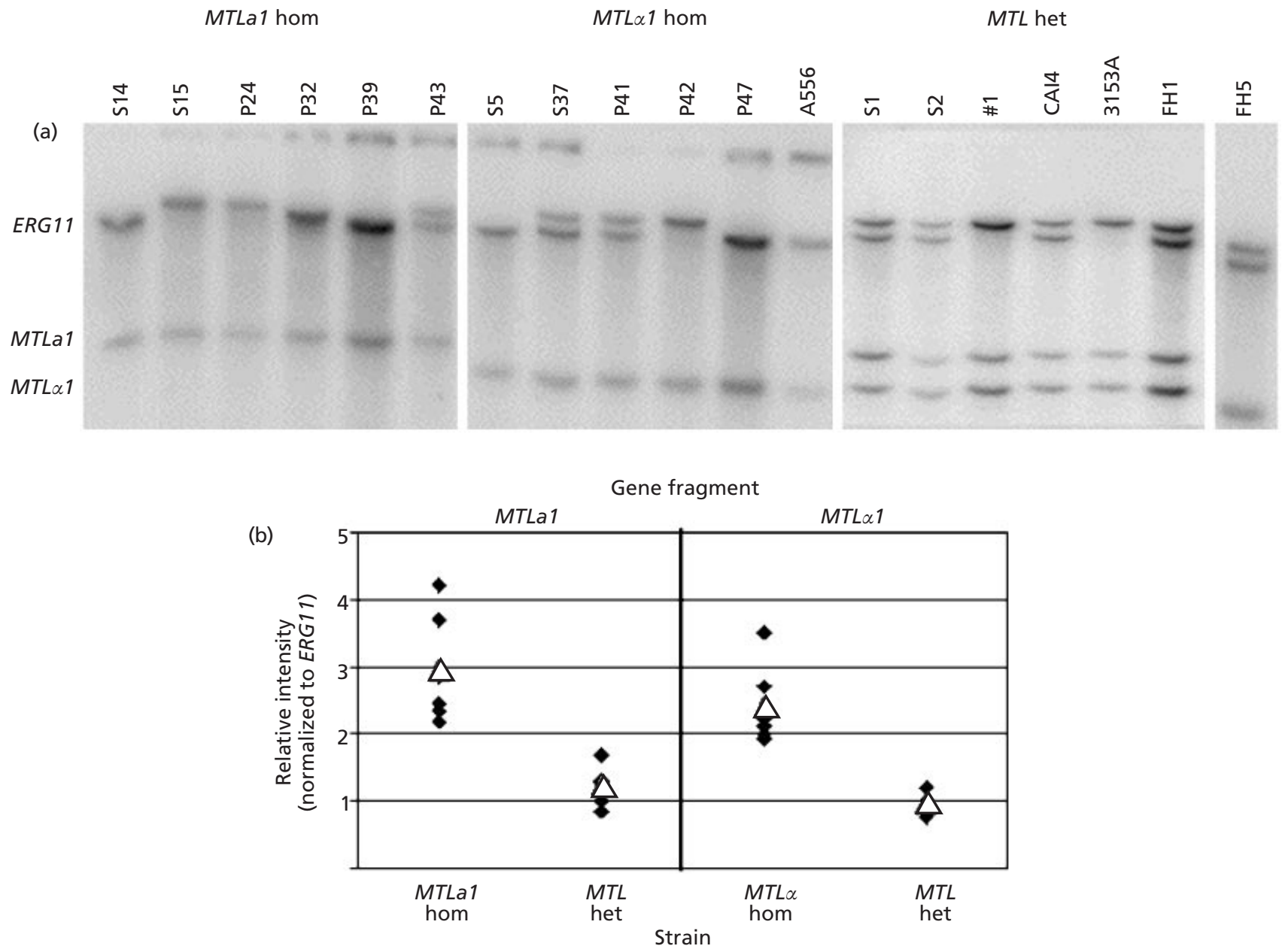

Fig. 6. (a) Southern blot of Haelll-digested genomic DNA probed with MTLa1-Erg11-MTL $\alpha 1$ triple probe. A probe containing $50 \mathrm{bp}$ of MTLa1, ERG11 and MTL $\alpha 1$ was constructed using PCR and radiolabelled. This triple probe was hybridized to a Southern blot of Haelll-digested genomic DNA. The probe hybridized to two ERG11 bands caused by restriction fragment polymorphism. Both ERG11 bands were present in FH1 and FH5. The MTLahom, MTLahom and FH5 sections are from three separate blots. (b) The intensity of these bands was quantified and the MTLa1 and MTL $\alpha 1$ bands were normalized to ERG11 for each isolate. Each diamond represents the mean intensity of a single isolate from three experiments. The triangles indicate the mean intensity of that group of isolates. On average, the MTLhom strains appear to carry at least two copies of either the MTLa or the MTL $\alpha$ locus.

a HaeIII restriction site. All bands were of the expected size predicted by HaeIII restriction maps of the published sequences (Table 1). The intensity of the MTL bands on three separate Northern blots was quantified and normalized to the total intensity of the ERG11 band(s). The scale was then adjusted so that the relative intensity of the CAI4 band was set to one. Although there was some variation around the mean, the relative intensities of the MTLa1 and MTL $\alpha 1$ bands of the MTLhom isolates were approximately twice the intensity of those bands in the control MTLhet strains (Fig. 6b). This indicates that there are two copies of the MTLa or $M T L \alpha$ locus in the MTLhom strains, suggesting that the MTLhom strains are the result of gene conversion or mitotic recombination that replaced one of the $M T L$ loci with a duplicate of the other. The presence of two ERG11 restriction fragments in some MTLhom strains, including isolates FH1 and FH5 from a serial set of isolates, suggests that the hypothetical gene conversion or mitotic recombination did not involve the ERG11 gene.

\section{Fluconazole susceptibility is unaltered in MTL locus disruptions}

It is possible that the MTL genes themselves have an effect on azole drug resistance. Gene disruptions of MTLa1, MTL $\alpha 1$ and MTL $\alpha 2$ have been previously described (Hull et al., 2000). In these gene disruptions URA3 was inserted using homologous recombination to disrupt the gene or locus of interest in the ura3 auxotrophic strain CAI4. The susceptibility of these isolates to fluconazole was measured using Etest strips (according to the manufacturer's specifications) and by 
Table 4. Fluconazole MICs and genotypes of strains with gene disruptions

\begin{tabular}{|c|c|c|}
\hline Strain & Genotype* & Fluconazole MIC† \\
\hline CAI4 & $\frac{M T L a}{M T L \alpha} \frac{u r a 3}{u r a 3}$ & $1 \cdot 00$ \\
\hline CHY 247 & $\frac{\text { mtla1::hisG-URA3-hisG }}{M T L \alpha} \frac{\text { ura3 }}{\text { ura3 }}$ & $0 \cdot 25$ \\
\hline CHY 257 & $\frac{m \text { tla } 1:: \text { his } G}{M T L \alpha} \frac{\text { ura } 3}{\text { ura3 }}$ & $0 \cdot 25$ \\
\hline CHY 477 & $\frac{m \text { tla } 1: \text { :his } G}{M T L \alpha} \frac{\text { ura } 3}{\text { ura3 }} \frac{\text { ade } 2: \text { :his } G-U R A 3-\text { his } G}{\text { ade2::his } G}$ & $0 \cdot 25$ \\
\hline CHY 420 & $\frac{m t l \alpha 1: \text { :hisG-URA3-hisG mtla2: :hisG }}{\text { MTLa }} \frac{\text { ura3 }}{\text { ura3 }}$ & $0 \cdot 25$ \\
\hline CHY 439 & $\frac{m t l \alpha 1:: \text { is } G \text { mtl } \alpha 2: \text { his } G}{\text { MTLa }} \frac{\text { ura3 }}{\text { ura3 }}$ & $0 \cdot 50$ \\
\hline CHY 484 & $\frac{\text { mtla locus: :his }}{\text { MTL } \alpha} \frac{\text { ura3 }}{\text { ura3 }} \frac{\text { ade2: :hisG-URA3-his } G}{\text { ade2::hisG }}$ & $0 \cdot 25$ \\
\hline CHY 486 & $\frac{\text { mtla locus: :his } G}{\text { MTLa }} \frac{\text { ura3 }}{\text { ura3 }} \frac{\text { ade2: :hisG-URA3-his } G}{\text { ade2::hisG }}$ & $0 \cdot 25$ \\
\hline
\end{tabular}

*All ura3 mutants are ura3::imm434. † MIC determined by NCCLS.

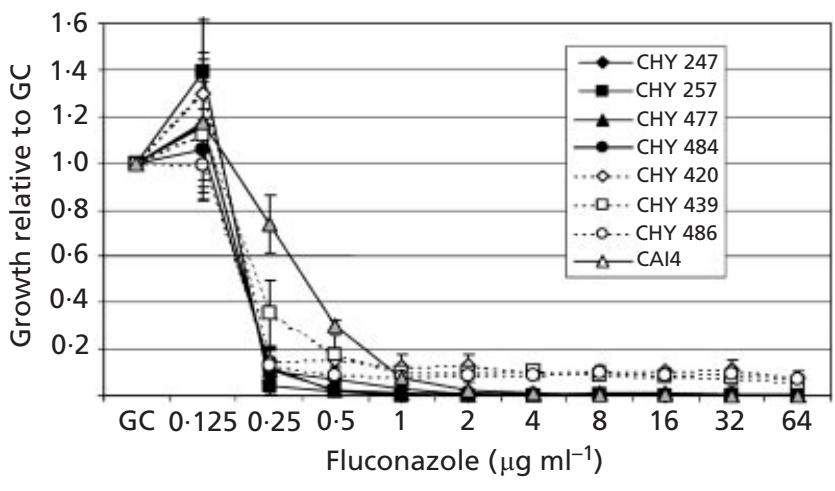

Fig. 7. Susceptibility of $M T L$ gene disruptions. The fluconazole MIC of strains with disruptions of the MTLa1 and MTL $\alpha 1$ genes and the MTLa and MTL $\alpha$ loci (see Table 4 for genotypes) were measured using the NCCLS M27A protocol. Although the disrupted strains tended to be more sensitive than the parent strain CAI4, the differences were not significant based on NCCLS guidelines. All strains lacking the MTL $\alpha 1$ gene were capable of limited growth up to the highest concentration of fluconazole tested. GC, no-drug control.

the standard NCCLS microbroth method (National Committee for Clinical Laboratory Standards, 1997). The URA3-disrupted strains and ura3 auxotrophic revertants of those strains displayed no change in susceptibility compared to the parental and wild-type strains when tested on Etest strips (Table 4). These results were verified using the NCCLS microdilution assay. However, in the NCCLS assays a subtle trend was visible (Fig. 7). All of the strains in which MTL $\alpha 1$ and $M T L \alpha 2$ had been deleted were able to grow a small amount, even at the highest levels of fluconazole. This sort of residual growth has previously been documented as a problem in determining macrodilution MIC endpoints, although the levels seen for the MTL $\alpha 1$ strains would not alter the MIC of the strains.

\section{DISCUSSION}

Drug resistance in pathogenic fungi is a serious threat to the growing number of immunocompromised patients. It is critical to understand how these opportunistic pathogens are able to circumvent current therapies in order to design effective new therapies and treatments. Previous work has shown that mutation or overexpression of the enzyme targeted by azole drugs, Erg11p, and overexpression of the membrane-bound pumps encoded by CDR1, CDR 2 and MDR 1 are correlated with increased resistance to azoles (White, 1997a). These mechanisms are present in only a portion of the azoleresistant isolates of C. albicans (Stevens \& White, 2000). In this study we have described a novel correlation between homozygosity at the C. albicans MTL loci and resistance to azole drugs.

The PCR screen of 96 clinical isolates clearly showed a relationship between MTL homozygosity and azole resistance (Table 3). Although the statistical relationship between drug resistance and MTL homozygosity is 
significant, the presence of the sensitive MTLhom isolate P24 suggests that MTL homozygosity alone is not sufficient for resistance. This conclusion was further supported by the unchanged susceptibility of the MTL gene disruptions (Fig. 7). These sensitive MTLhom strains may be independent of resistance, or missing functional genes necessary for $M T L$-related resistance, or are starting to develop resistance.

The results of the PCR screen were confirmed by additional PCR screens of other genes in the MTL loci and by the hybridization of radiolabelled MTLa1 and MTL $\alpha 1$ genes to CHEF Southern blots (Fig. 3). The MTLa1 and MTL $\alpha 1$ probes hybridized to chromosomes other than chr 5 in some of the MTLhom isolates. These extra copies were present in only a few of the MTLhom isolates and are therefore unlikely to be related to increased azole resistance in these isolates.

Further evidence of a correlation between MTL homozygosity and azole resistance comes from the corresponding increase in azole resistance seen after loss of one of the MTL loci in the FH series of isolates (Fig. 4). The final isolate was taken from post-mortem lung tissue, and thus was likely protected from fluconazole selection pressure that appears to have caused the other isolates to develop resistance, and lose the MTLa locus (Marr et al., 1997). The gain of resistance in this series of isolates has been previously correlated with upregulation of CDR1. However in three of the five MTLhom isolates in which $C D R 1$ levels have been analysed no upregulation of CDR1 is seen (data not shown). In fact, no known resistance mechanism is present in all of the five resistant MTLhom isolates for which we have data.

The FH series of isolates has also been shown to have a heterogeneous resistance (HetR) phenotype (Marr et al., 2001). A fraction of c.f.u. from HetR strains are able to grow on plates containing fluconazole, but the resulting colonies retain both azole susceptibility and the HetR phenotype. Preliminary experiments on FH1 colonies from azole-containing plates show no loss of heterozygosity at the MTL locus.

The multiple copies of chr 5 indicated by the presence of heterozygous SNPs on that chromosome (Table 2) suggest that the loss of MTL homozyosity is not due to a loss of one copy of that chromosome. Furthermore, duplicate copies of the MTL locus (Fig. 6b), and heterozygous SNPs on chr 5 (Table 2) suggest that the absence of one of the MTL loci is not due to deletion or chromosome loss and duplication. Given that the SNPs from chr 5 are in non-coding regions, and thus have no selection advantage, it is unlikely that these polymorphisms arose in the short time between a theoretical loss and subsequent duplication of chr 5. Homozygosity at the MTL locus could also be achieved through a gene conversion or homologous recombination that replaced the MTLa locus with a second copy of the MTL $\alpha$ locus or the reverse. Although this loss of heterogzygosity extends through the MTL locus, the presence of heterozygous restriction fragments in ERG11 in the FH series both before and after heterozygosity was lost at the MTL locus (Fig. 6a) suggests that this region does not include ERG11. Mapping the size of the region of lost heterozygosity will distinguish between those two possibilities and will limit the list of genes potentially responsible for this correlation.

Changes in chromosome copy number have been linked to various morphological mutants, growth in L-sorbose and alterations in carbon and nitrogen utilization (Janbon et al., 1998, 1999; Rustchenko et al., 1994). Changes in copy number of chr 4 (monosomy) and chr 3 (trisomy) have been associated with azole resistance (Perepnikhatka et al., 1999). However, changes in copy number of chr 5 have not previously been associated with drug resistance. This study has focused on the MTL locus on chr 5, which remains disomic in these isolates. Therefore, the changes observed at the MTL locus are unlikely to be related to the changes in chromosome number observed above.

Our results suggest a number of genes that could be responsible for a correlation between MTL homozygosity and azole resistance. These possibilities can be split into two categories. The first category consists of genes that cause slight resistance when mutated in one allele and much greater resistance when the mutation is in both alleles. Selective pressure would then favour replacement of the wild-type allele with a mutant allele in a population of $C$. albicans grown in the presence of azole drugs. Any gene within the region of lost heterozygosity that is present in two copies could potentially fall into this category. ERG11 is the obvious example of this sort of selection, as it is the only gene related to azole resistance that lies on chr 5 , and previous experiments have shown that gene conversion does occur following a point mutation in one allele of ERG11 (White, 1997b; Franz et al., 1998). However, the presence of heterozygous SNPs in the ERG11 coding region and terminator in 5 of the 12 MTLhom suggests that there has been no recent loss of heterozygosity in ERG11 in these isolates and therefore that ERG11 is not the gene responsible for the correlation between MTL homozygosity and resistance. Other genes that could potentially fall into this category include the $O B P$ and $P I K$ genes in the MTL loci. Homologues of the OBP genes in particular have been shown to play a role in ergosterol homeostasis in S. cerevisiae (Beh et al., 2001).

The other category of possibilities is a general resistance effect that results from the loss of one of the MTL specific genes. Of particular interest are the MTLa1 and $M T L \alpha 2$ genes. The homologues of the products of these genes in $S$. cerevisiae form a heterodimeric transcriptional regulator (Herskowitz et al., 1997). It therefore follows that if a functionally similar heterodimer forms in C. albicans, loss of one of those genes would lead to altered levels of a number of genes, possibly including those involved in azole resistance. Although the gene disruptions of the MTL loci caused little change in azole susceptibility (Table 4), the laboratory strain in which the gene disruptions were performed may have a 
nonfunctional copy of a gene that contributes to resistance.

Future experiments will determine which of these models is correct. These experiments include characterizing the MTLhom strains further and determining the mechanism by which they develop resistance decreased import, increased export, increased resistance of the Erg11p in these isolates, or some other undescribed mechanism.

\section{ACKNOWLEDGEMENTS}

We thank Drs Raleigh Bowden and Kieren Marr (Fred Hutchinson Cancer Research Center, Seattle, WA, USA) for the use of their clinical isolates. We thank Drs Christina Hull and Alexander Johnson for the use of their MTL gene disruption strains. We thank members of the White and Marr laboratories for their support and comments on this manuscript. This research was supported by NIH NIDR grants RO1 DE-11367 and RO1 DE-14161. T.C. W. is the recipient of a New Investigator Award in Molecular Pathogenic Mycology from the Burroughs Wellcome Fund.

\section{REFERENCES}

Beh, C. T., Cool, L., Phillips, J. \& Rine, J. (2001). Overlapping functions of the yeast oxysterol-binding protein homologues. Genetics 157, 1117-1140.

Cowen, L. E., Sirjusingh, C., Summerbell, R. C., Walmsley, S., Richardson, S., Kohn, L. M. \& Anderson, J. B. (1999). Multilocus genotypes and DNA fingerprints do not predict variation in azole resistance among clinical isolates of Candida albicans. Antimicrob Agents Chemother 43, 2930-2938.

Fonzi, W. A. \& Irwin, M. Y. (1993). Isogenic strain construction and gene mapping in Candida albicans. Genetics 134, 717-728.

Franz, R., Kelly, S. L., Lamb, D. C., Kelly, D. E., Ruhnke, M. \& Morschhauser, J. (1998). Multiple molecular mechanisms contribute to a stepwise development of fluconazole resistance in clinical Candida albicans strains. Antimicrob Agents Chemother 42, 3065-3072.

Georgopapadakou, N. H. \& Walsh, T. J. (1996). Antifungal agents - chemotherapeutic targets and immunologic strategies. Antimicrob Agents Chemother 40, 279-291.

Herskowitz, I., Rine, J. \& Strathern, J. (1997). Mating-type determination and mating-type interconversion in Saccharomyces cerevisiae. In The Molecular and Cellular Biology of the Yeast Sacharomyces, pp. 583-596. Edited by J. Pringle, J. Broach \& E. Jones. Plainview, NY: Cold Spring Harbor Laboratory.

Hull, C. M. \& Johnson, A. D. (1999). Identification of a mating type-like locus in the asexual pathogenic yeast Candida albicans. Science 285, 1271-1275.

Hull, C. M., Raisner, R. M. \& Johnson, A. D. (2000). Evidence for mating of the "asexual" yeast Candida albicans in a mammalian host. Science 289, 307-310.

Janbon, G., Sherman, F. \& Rustchenko, E. (1998). Monosomy of a specific chromosome determines L-sorbose utilization: a novel regulatory mechanism in Candida albicans. Proc Natl Acad Sci U S A 95, 5150-5155.

Janbon, G., Sherman, F. \& Rustchenko, E. (1999). Appearance and properties of L-sorbose-utilizing mutants of Candida albicans obtained on a selective plate. Genetics 153, 653-664.

Johnson, A. D. (1995). Molecular mechanisms of cell-type determination in budding yeast. Curr Opin Genet Dev 5, 552-558.
Lai, M. H. \& Kirsch, D. R. (1989). Nucleotide sequence of cytochrome P450 L1A1 (lanosterol 14 alpha-demethylase) from Candida albicans. Nucleic Acids Res 17, 804.

Law, D., Moore, C. B., Wardle, H. M., Ganguli, L. A., Keaney, M. G. \& Denning, D. W. (1994). High prevalence of antifungal resistance in Candida spp. from patients with AIDS. J Antimicrob Chemother 34, 659-668.

Lott, T. J. \& Effat, M. M. (2001). Evidence for a more recently evolved clade within a Candida albicans North American population. Microbiology 147, 1687-1692.

Magee, B. B. \& Magee, P. T. (2000). Induction of mating in Candida albicans by construction of MTLa and MTL $\alpha$ strains. Science 289, 310-313.

Marr, K. A., White, T. C., van Burik, J. A. H. \& Bowden, R. A. (1997). Development of fluconazole resistance in Candida albicans causing disseminated infection in a patient undergoing marrow transplantation. Clin Infect Dis 25, 908-910.

Marr, K. A., Lyons, C. N., Rustad, T. R., Bowden, R. A. \& White, T. C. (1998). Rapid, transient fluconazole resistance in Candida albicans is associated with increased mRNA levels of CDR. Antimicrob Agents Chemother 42, 2584-2589.

Marr, K. A., Lyons, C. N., Ha, K., Rustad, T. R. \& White, T. C. (2001). Inducible azole resistance associated with a heterogeneous phenotype in Candida albicans. Antimicrob Agents Chemother 45, 52-59.

National Committee for Clinical Laboratory Standards (1997). Reference method for broth dilution antifungal susceptibility testing of yeasts. Approved Standard. NCCLS document M27-A. Wayne, PA: National Committee for Clinical Laboratory Standards.

Odds, F. C. (1988). Candida and Candidosis: a Review and Bibliography. London: Baillière Tindall.

Perepnikhatka, V., Fischer, F. J., Niimi, M., Baker, R. A., Cannon, R. D., Wang, Y. K., Sherman, F. \& Rustchenko, E. (1999). Specific chromosome alterations in fluconazole-resistant mutants of Candida albicans. J Bacteriol 181, 4041-4049.

Pfaller, M. A., Jones, R. N., Doern, G. V., Sader, H. S., Hollis, R. J. \& Messer, S. A. (1998). International surveillance of bloodstream infections due to Candida species: frequency of occurrence and antifungal susceptibilities of isolates collected in 1997 in the United States, Canada, and South America for the SENTRY program. J Clin Microbiol 36, 1886-1889.

Rustchenko, E. P., Howard, D. H. \& Sherman, F. (1994). Chromosomal alterations of Candida albicans are associated with the gain and loss of assimilating functions. J Bacteriol 176, 3231-3241.

Sambrook, J., Fritsch, E. F. \& Maniatis, T. (1989). Molecular Cloning: a Laboratory Manual, 2nd edn. Cold Spring Harbor, NY : Cold Spring Harbor Laboratory.

Stevens, D. A. \& White, T. C. (2000). Frequency of known resistance mechanisms in clinical C. albicans isolates resistant or susceptible to fluconazole. In ICAAC 2000, Abstract 1952. Toronto, Canada.

Uitenbroek, D. G. (1997). SISA Fisher Exact. http://home.clara. net/sisa/fisher.htm.

White, T. C. (1997a). Increased mRNA levels of ERG16, CDR, and MDR1 correlate with increases in azole resistance in Candida albicans isolates from a patient infected with human immunodeficiency virus. Antimicrob Agents Chemother 41, 1482-1487.

White, T. C. (1997b). The presence of an R467K amino acid substitution and loss of allelic variation correlate with an azoleresistant lanosterol 14 alpha demethylase in Candida albicans. Antimicrob Agents Chemother 41, 1488-1494. 
White, T. C., Pfaller, M. A., Rinaldi, R. G., Smith, J. \& Redding, S. W. (1997). Stable azole drug resistance associated with a substrain of Candida albicans from an HIV-infected patient. Oral Dis 3, S102-S109.

White, T. C., Marr, K. A. \& Bowden, R. A. (1998). Clinical, cellular, and molecular factors that contribute to antifungal drug resistance. Clin Microbiol Rev 11, 382-402.
Wright, W. L. \& Wenzel, R.P. (1997). Nosocomial Candida. Epidemiology, transmission, and prevention. Infect Dis Clin $\mathrm{N}$ Am 11, 411-425.

Received 8 October 2001; revised 7 December 2001; accepted 14 December 2001. 